\title{
Calixarenes: paradoxes and paradigms in molecular baskets
}

\author{
C. David Gutsche, Janet S. Rogers, Donald Stewart, and Keat-Aun See
}

Department of Chemistry, Washington University, St Louis, MO 63130, USA

Calixarenes are $\left[1_{n}\right]$ metacyclophanes that can be prepared in one-flask syntheses from cerain p-alkylphenols and formaldehyde. Their history is a facet of phenol-formaldehyde chemistry which had its genesis in the nineteenth century laboratories of Adolph von Baeyer. Their structures were partially elucidated by Zinke and by Niederl in the 1940's, but incomplete data concerning their synthesis and purification created troubling paradoxes. As a result of the research by groups in Mainz, Parma, and St Louis in the late 1970's and early 1980's the paradoxes have been resolved and a paradigm has been created that has elicited widespread and increasing attention to these compounds.

The paradigm, which states that cyclic oligomers of various sizes are formed from the condensation of certain $p$-substituted phenols and formaldehyde, provides the guideline for easily obtaining $p$-tert-butylcalix [4], calix[6], and calix[8]arenes in a single step. The $\mathrm{AlCl}_{3}$ catalyzed removal of the tert-butyl groups then makes possible the introduction of a variety of substituents, and several methods have been developed for this purpose.

The conformational mobility of the calixarenes, which played a key role in the aforementioned paradox, has been well-studied both in the solid state and in solution. It can often be curtailed by appropriate substitution on the phenolic oxygen atoms which freezes the calixarene into a particular conformation.

Calixarenes are especially atractive as complexing agents and enzyme mimics, because their basket-like shape allows them to act as hosts to guest molecules of appropriate size and character. As the result of the ease with which calixarenes can now be synthesized, functionalized, and conformationally fixed much effort is currently being devoted to such studies.

Webster's dictionary defines paradox as "a tenet contrary to received opinion" and paradigm simply as "a model or pattern". Thomas Kuhn, a well known philosopher and historian of science, has given paradigm a broader and in some respects a less well defined meaning ${ }^{1}$. A scientific paradigm, he says, is created by an achievement that is sufficiently unprecedented to attract an enduring group of adherents away from competing modes of scientific activity and is sufficiently open ended to leave all sorts of problems for the redefined group of practitioners to resolve. It is Kuhn's thesis that the interplay of paradoxes and paradigms is responsible for what may seem to be an orderly progression in science but that in actual fact is a succession of fits and starts.

Brief History of the Calixarenes: The present work concerns a set of paradigms that had their inception over a century ago in the laboratories of Adolf von Baeyer ${ }^{2}$. Baeyer was fascinated with colored compounds, and one of the types of reaction that he studied in his quest for new dyes was that of various phenols with aldehydes and ketones. In some cases he was successful in isolating and identifying the products, but when he took the simplest ingredients - namely, phenol itself and formaldehyde - he got a dark colored resinous tar that he despaired of characterizing and simply dispatched to the waste jar.

Not until early in the twentieth century was this reaction finally tamed by Leo Baekeland who discovered that by using a small and controlled amount of base in the condensation of phenol and formaldehyde a commercially appealing material could be obtained. In 1907 he filed a patent ${ }^{3}$ on this process for making the material he called Bakelite. The age of plastics had begun, and the first paradigm in this story was in place. The Bakelite process is viewed as a legitimate paradigm because it satisfies both of Kuhn's criteria. It was an unprecedented achievement that attracted many adherents, and it inspired a great deal of research aimed at altering and improving the product.

The second event in this story took place at the Petrolite Company a half century later. Petrolite, located in the St Louis area, manufactures demulsifiers for crude oil. In the 1950 's it marketed a material that was made by the condensation of a $p$-alkylphenol with formaldehyde followed by treatment of the condensate with ethylene oxide to produce an oxyalkylate that was thought to have a linear oligomeric structure. Shortly after marketing this material the company received complaints both from the customers and from the workers in the manufacturing plant that 
messy sludges were precipitating from the hydrocarbon solution. Not knowing the cause, the manufacturing plant appealed to the research group for help, and the problem was turned over to John Munch who devised a laboratory simulation of the plant procedure. It consisted simply of mixing paraformaldehyde with a $p$-alkylphenol such as $p$ octylphenol in xylene, adding a small amount of sodium hydroxide, and refluxing the mixture for several hours ${ }^{4}$, a recipe that is now generally referred to as the "Petrolite Procedure". Precipitating from the cooled reaction mixture was a copious amount of a colorless, very high melting, very insoluble material that seemed to explain the sludges about which the customers were complaining.

The third event in this story actually occurred prior to the one at Petrolite. In the Bakelite process the phenol and formaldehyde are first condensed to a viscous mass, and this viscous mass is then heated to produce the hard brittle condensate, this second step being called the"curing" phase. Exactly what goes on during the curing phase is still not completely understood, although it has been the object of study almost since the day that Baekeland started selling his product. One such study was carried out in the 1940's by an Austrian chemist named Alois Zinke at the University of Graz. Zinke and his coworker Eric Ziegler used p-alkylphenols instead of phenol itself, correctly reasoning that this should reduce the amount of cross linking in the condensate and might thereby simplify the investigation of the chemistry of the curing reaction. When they mixed $p$-tert-butylphenol and formaldehyde, added sodium hydroxide, and heated the mixture in linseed oil at a temperature higher than $200^{\circ} \mathrm{C}$ they obtained a very high melting, organic solvent-insoluble material to which at first they demured from assigning a structure 5 because the cryoscopic molecular weight seemed to correspond to that of an octamer, which seemed unlikely. Three years later, perhaps bolstered by the cyclic tetrameric structure that Joseph Niederl had suggested for the condensation product from resorcinol and acetaldehyde ${ }^{6}$, Zinke assigned the cyclic tetrameric structure shown at the right to his compounds ${ }^{7}$. Thus, another paradigm was born. It said that $p$-substituted phenols condense with formaldehyde in the presence of inorganic base to form cyclic tetramers, and it was this paradigm that was revealed to the Petrolite researchers when, after having carried out their simulated plant procedure which produced the copious precipitate, they searched the literature and discovered Zinke's publications. At this point the BaekelandBakelite paradigm, the Zinke cyclic tetramer paradigm, and the chemistry at the Petrolite Company came into convergence.

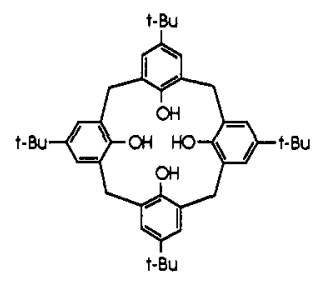

p-fert-Butylcalix(4)arene

As a consultant to the Petrolite Company the senior author knew of this convergence of paradigms and saw it as an entry into the molecular baskets that were desired for the construction of enzyme mimics. A space filling molecular model of a Zinke cyclic tetramer shows that it is cup- or vase-shaped, and because of the resemblance of

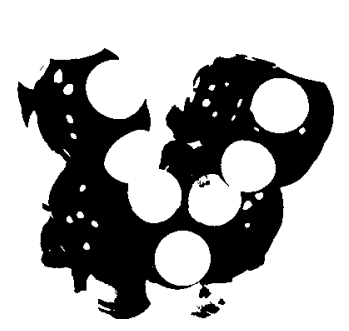

this shape to that of a kind of Greek vase called a calix crater the name calixarene was coined ${ }^{8}$, calix defining its shape and arene indicating the presence of aryl groups. To specify this particular calixarene as a cyclic tetramer a bracketed [4] is placed between calix and arene - thus, calix[4]arene.

At first,the Zinke paradigm stating that cyclic tetramers are obtained from the condensation of $p$-substituted phenols and formaldehyde appeared to hold true, promising the facile synthesis of dozens of interesting calixarenes. Upon closer inspection, however, flaws emerged. The products were determined not to be single components but mixtures, a fact that Sir John Cornforth ${ }^{9}$ had already discovered fifteen years earlier when he had repeated the Zinke experiment. But, the formation of more than one compound presents a paradox. If the Zinke reaction produces only cyclic tetramers and if more than one product is obtained, what are the structures of the products? Cornforth answered this by retaining the Zinke paradigm but modifying it by postulating the existence of what today we call conformational isomers. He recognized that four different orientations of the aryl rings in the cyclic tetramer are possible, leading to structures which have subsequently been named as cone, partial cone, 1,2-alternate, and 1,3-alternate conformers ${ }^{10}$.

One of the most powerful destroyers of paradigms is a new instrumental technique. The telescope and the Copernican revolution, the microscope and the biological revolution, the cyclotron and the nuclear revolution are but a few of the many examples of the tremendous impact that new instruments can have on existing concepts. In the case of the Zinke paradigm as modified by Cornforth the instrument of destruction was the NMR spectrometer. In the early 1970's, concomitant with early work on the calixarenes at Washington University, a group in Germany headed by Hermann Kämmerer was carrying out the syntheses of calixarenes by an unequivocal stepwise route which produced authentic cyclic tetramers. When Kämmerer measured the proton NMR spectrum of these materials ${ }^{11}$ the spectral patterns were found to be different at low and high temperatures. At a temperature of $20^{\circ} \mathrm{C} \mathrm{a}$ pair of doublet resonances was observed for the hydrogens attached to the bridging methylene groups, whereas at $60^{\circ} \mathrm{C}$ only a single line was observed. The most reasonable interpretation for this behavior is to postulate that at $60^{\circ}$ $\mathrm{C}$ the calixarene is undergoing a cone to cone transformation at a rate that is rapid on the NMR time scale, whereas at $20^{\circ} \mathrm{C}$ this interconversion is slow on the NMR time scale. But, even at $20^{\circ} \mathrm{C}$ the "slow" rate is far too fast to allow isolation and separation of individual conformers on a "laboratory" time scale. The cyclic tetramer is conformationally mobile, not rigid as Cornforth postulated as the basis for explaining the mixtures that are produced in the Zinke reaction. Cone, partial cone, inverted cone conformers are all in rapid equilibrium with one another, with the cone conformers being the dominant species.

This creates still another paradox. If the components of the mixture from a Zinke reaction aren't conformational isomers, what are they? Research by the Washington University group uncovered the answer to this paradox, the 
first clue being provided by the treatment of the material obtained by the Petrolite Procedure with a limiting amount of 2,4-dinitrochloro-benzene ${ }^{12}$. The resulting product was a mixture in which one of the compounds had a ratio between functionalized and unfunctionalized $\mathrm{OH}$ groups of 1:3, another of the compounds had a ratio of 3:1 and a third compound had a ratio of 1:7. The first two of these ratios are interpretable on the basis of a cyclic tetramer, but the $1: 7$ ratio is commensurate only with a cyclic octamer. That this was a correct interpretation was subsequently verified in a variety of ways osmometric molecular weight, mass spectral molecular weight, and ultimately by X-ray crystallography 13 (see structure at right). Thus. the Zinke paradigm that says that cyclic tetramers are formed from $p$-substituted phenols and formaldehyde had to be replaced by a new paradigm that says that cyclic oligomers of various sizes are formed from these reactants.

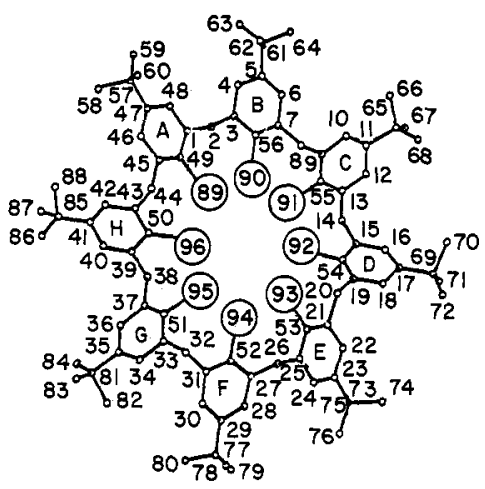

Mechanism of the Calixarene-Forming Reaction: With the new paradigm came new questions, the most challenging of which concerns the mechanism of the calixarene-forming reaction. A prevailing paradigm based on the frequent occurrence in nature of cyclic compounds such as porphyrins asserts that cyclic tetramers are the structures that are most likely to be formed in many cyclization processes. It was obeisance to this paradigm that guided Zinke, Niederl, Cornforth, and others in their assignments of structure. Molecular models as well as experiments described below show that calix[3]arenes are too strained to be readily formed, and entropy considerations would seem to rule our larger cyclic oligomers such as calix[8]arenes. Yet, under the conditions of the Petrolite Procedure the cyclic octamer is formed in high yield and in a state of almost $100 \%$ purity. The current working hypotheses to explain this and other aspects of the calixarene-forming reaction are described below.

$p$-tert-Butylphenol and formaldehyde condense to form a mixture of linear oligomers - linear dimers, trimers, tetramers, etc- that are mono-hydroxymethylated and/or di-hydroxymethylated. The linear tetramer, which is

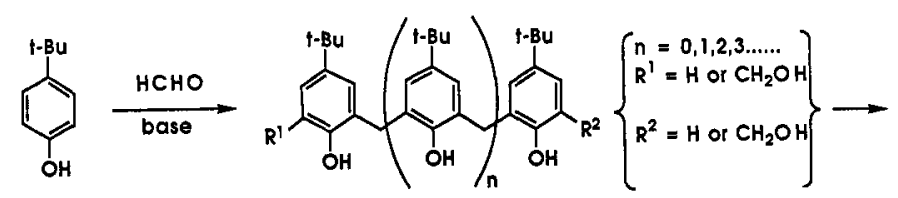
assumed to play a critical role, can exist in a variety of conformations, including ones that are designated as the crescent and the zig-zag forms. Both of these are stabilized by intramolecular hydrogen bonding.

X-Ray crystallographic data show that in the solid state the tetramer exists in the zig-zag conformation ${ }^{14}$, ana 11 is reasonable to assume that this reflects the preferred state in solution as well. For the linear tetramer to become a cyclic tetramer it is necessary that the zig-zag conformer first be converted to the crescent conformer, and this requires a certain amount of energy. Furthermore, if the linear tetramer is di-hydroxymethylated it must lose a molecule of formaldehyde as well as a molecule of water to form the calix [4]arene. There are two alternative events that appear more likely to occur. One is the loss of water from a di-hydroxymethylated tetramer to form the dihomooxacalix[4]arene, and this compound does, indeed, appear at an early stage of the reaction, diminishing in amount as the reaction proceeds to completion. It is viewed not as a direct precursor to the calixarenes but as a storage depot that provides calixarene-forming entities.

A second alternative pathway involves intermolecular association, through hydrogen bonding, of a pair of zigzag conformers to form what we have called a hemicalix[8]arene. The resulting hemicalix[8]arene is in a conformation that is remarkably close to the one that is known to characterize the cyclic octamer itself when it is in the solid state ${ }^{13}$. Thus, the hemicalix [8]arene has merely to clip together at the two ends of each of its linear tetramer components to form the cyclic octamer. Good experimental evidence has been adduced in support of the formation of the cyclic octamer before the cyclic tetramer, the latter appearing only when sufficiently strenuous reaction conditions are employed. If the conditions are sufficiently strenuous - that is, boiling diphenyl ether at $\mathrm{ca} 220^{\circ} \mathrm{C}$ the cyclic octamer is transformed to the cyclic tetramer. It is the present postulate that this proceeds as a kind of molecular mitosis 15 in which the cyclic octamer pinches together at two points across its ring and then splits into a pair of tetramers.

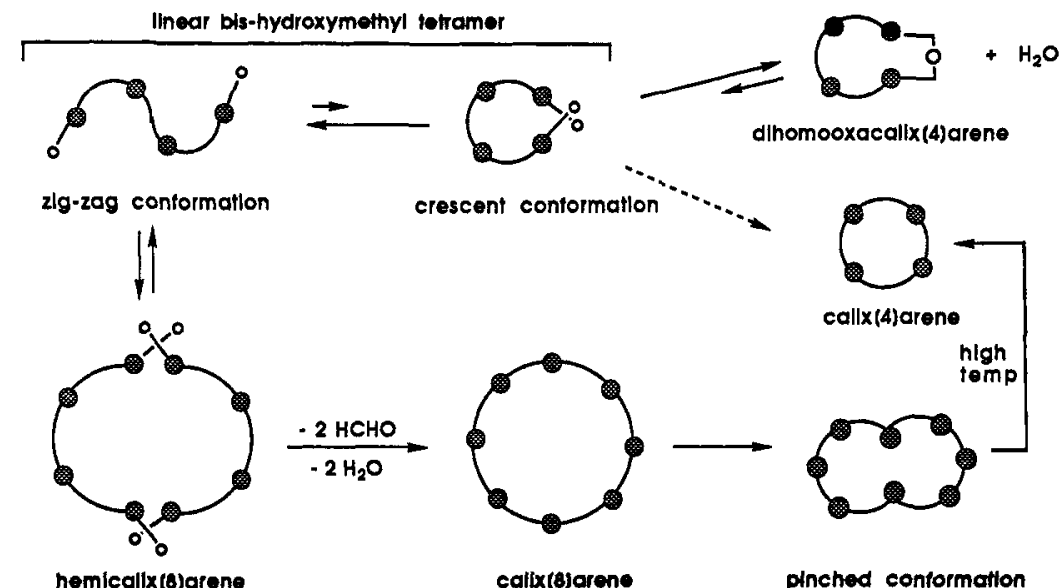


Emerging out of the paradoxes and paradigms described above has been a set of procedures that now make it possible to produce the cyclic tetramer, cyclic hexamer, and cyclic octamer in essentially unlimited quantity. These are perhaps the world's most accessible molecular baskets, and numerous research groups throughout the world are now busily capitalizing on this fact by exploring various facets of the chemistry of these compounds. Also available via this one-step process, although with much greater difficulty, are the odd numbered calixarenes - the cyclic pentamer and the cyclic heptamer as well as some even larger calixarenes. A careful analysis of crude calixarene mixtures, making use of conventional as well as HPLC analysis and separation techniques reveals an even more complex mixture than was originally realized. The table below lists some of the characteristics of the calixarenes that to date have been isolated and characterized, or simply detected.

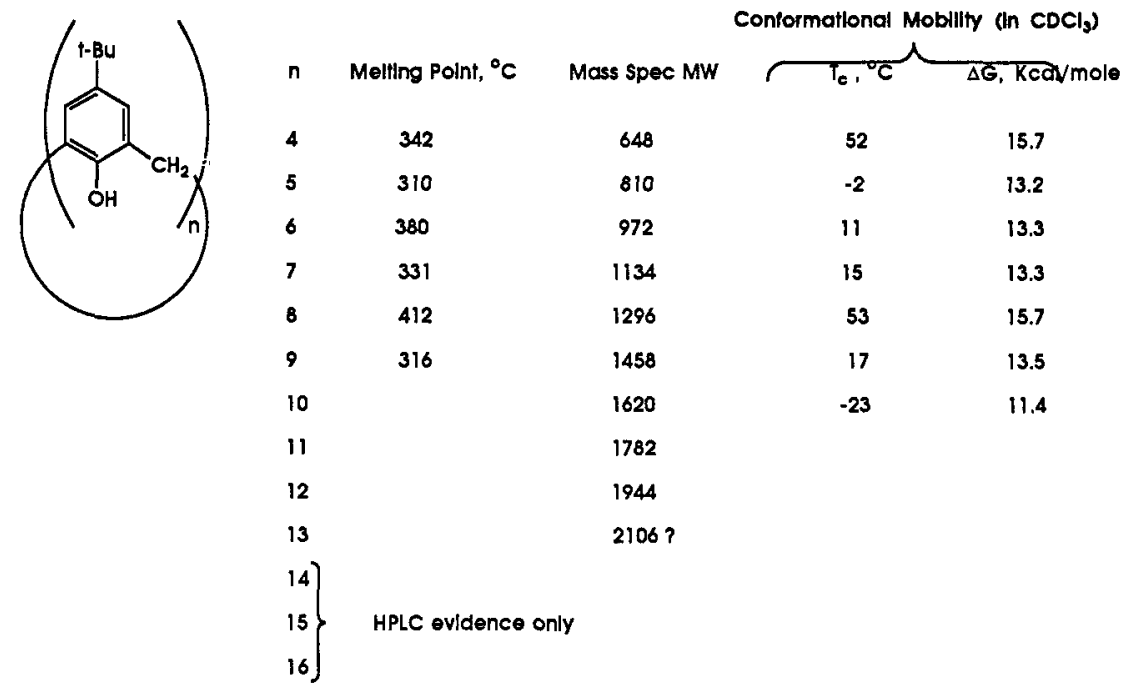

Normal science, says Thomas Kuhn, is what most scientists spend most of their lives doing. He characterizes it as puzzle solving according to the rules established by the prevailing paradigms, and this is what calixarene research in the 1980's has involved. Starting with calixarenes made in the ways described above, methods have been worked out for contouring their shapes, procedures for placing a variety of functional groups on their cavities have been devised, and their complexation and catalytic abilities have been explored. Some of the recent results from the study of calixarene chemistry in the Washington University laboratories are outlined below.

Oxacalixarenes: Dihomooxacalix[4]arene is known to be a major component in the early stages of calixarene forming reactions, and in some cases it can be isolated from the ultimate reaction mixture. It is likely that other oxacalixarenes are also formed, such as those shown below which are the tentatively assigned structures to the compounds isolated from the thermal dehydration of the bis-hydroxymethyl linear dimer.

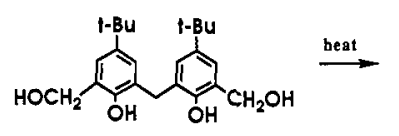

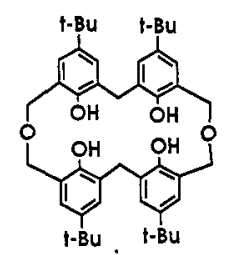

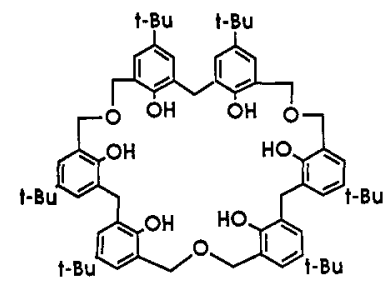

Calix[3]arenes: Moshfegh, Hakimelahi and coworkers ${ }^{16}$ have reported the synthesis of a calix[3]arene by the acid-catalyzed condensation of a linear dimer with a bis-hydroxymethylphenol. Attempts to repeat this synthesis have failed, and application of the Hayes and Hunter procedure ${ }^{17}$ to the monohydroxymethyl linear trimer, which would be expected to be even more likely to yield a calix[3]arene, gives the calix[6]arene as the major product. However, an HPLC analysis of the crude product shows weak signals at a position that might correspond to a calix[3]arene.

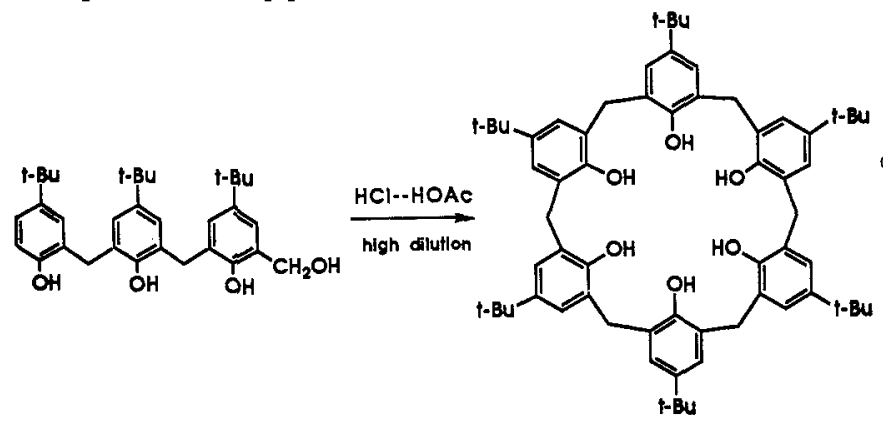

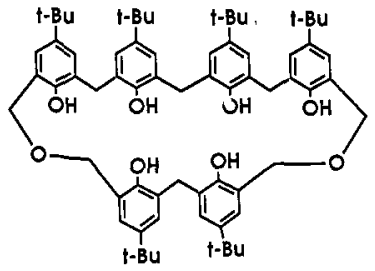

in<smiles>[TeH4]</smiles> 
Synthesis of a 1,2-Alternate Conformer: Numerous examples of calix[4]arenes frozen, by derivatization of the $\mathrm{OH}$ groups, into the cone, partial cone, or 1,3-alternate conformation are known, but only one example of a 1,2-alternate conformer has been published ${ }^{18}$, viz. the complex obtained by treatment of the tetramethyl ether of $p$ tert-butylcalix[4]arene with $\mathrm{Me}_{3} \mathrm{Al}$. The tetra-thioureido derivative of $p$-tert-butylcalix[4]arene is now shown by $\mathrm{X}$ ray crystallography to constitute a second example of a 1,2-alternate conformer.

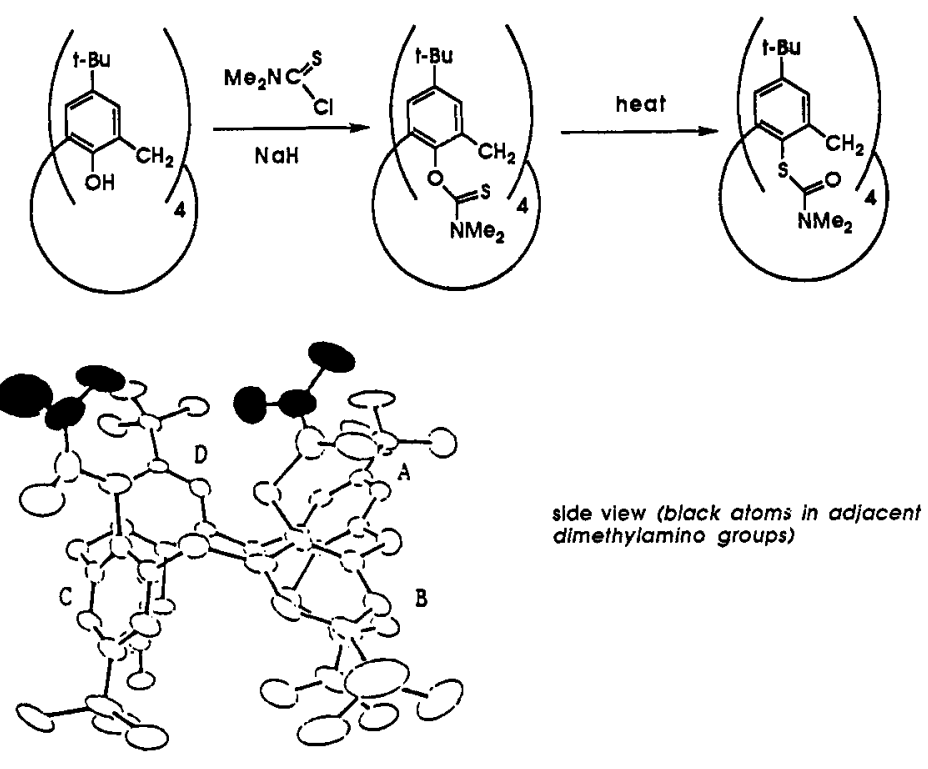

Partial Esterification of Calix[4]arenes: Methods are known for preparing the tetra-esters, tri-esters, and di-esters of calix[4]arenes, but mono-esters have been difficult to obtain. A procedure that now makes these available involves treatment of a calix[4]arene with 3,5-dinitrobenzoyl chloride to form the 1,3-diester in

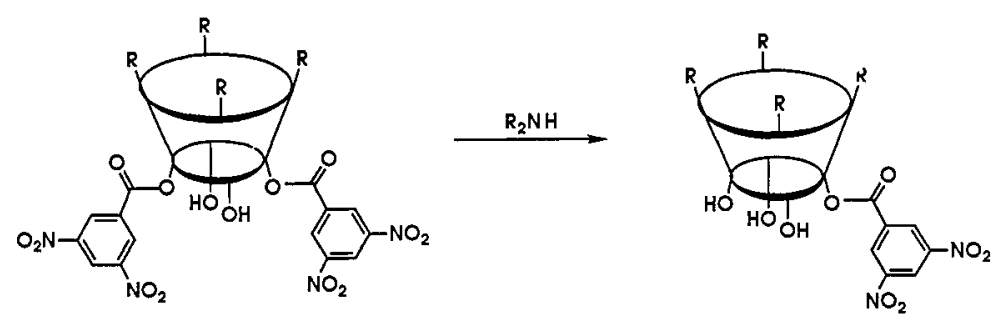

good yield followed by treatment with an organic base such as imidazole. The first ester group is removed at a much greater rate than the second, permitting easy isolation and purification of the mono-ester. A possible mechanism for the cleavage is depicted below in a scheme which recognizes the demonstrated necessity for a free phenolic function and the probable dependence on two equivalents of amine.

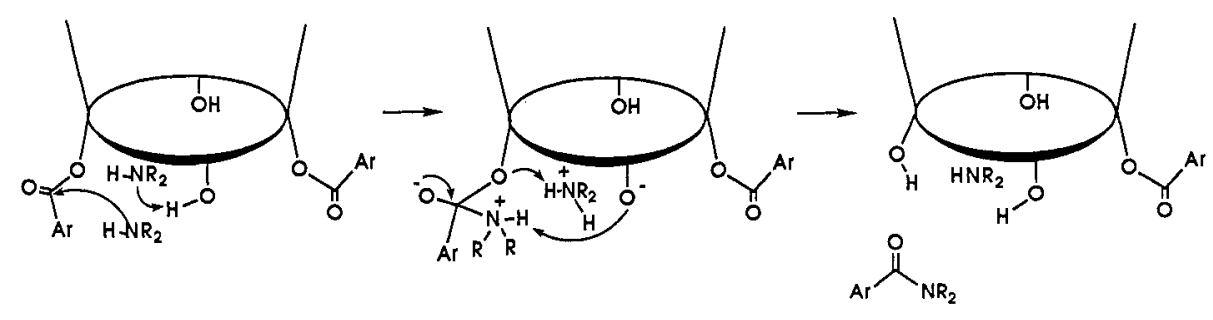

Partial Esterification of Calix[6]arenes: Treatment of p-tert-butylcalix[6]arenes with 3,5-dinitrobenzoy] chloride yields, instead of the anticipated 1,3,5-tri-ester, a mixture of 1,2,4,5-tetra-ester as the major product and penta-ester as the minor product. The structure and conformation of the 1,2,4,5-tetra-ester have been established by elemental analyses, FAB mass spectra, ${ }^{1} \mathrm{H}$ NMR, 2D NOE, and transient NOE measurements. It is concluded that the compound exists in a 1,2,3-alternate conformation in which two of the tert-butyl groups are tipped inward to fill the top and bottom cavities of the molecule, thus precluding effective complexation with external guest molecules. 
Designation of the H-atoms In the tetra-ester

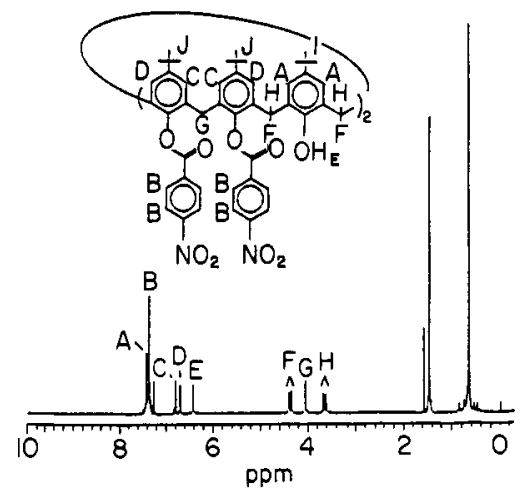

2-D NOE Measurements of the tefra-ester

$\begin{array}{ccccc} & A & B & D & E \\ A & - & -11.3 & 4.8 & 1.3 \\ B & -8.5 & - & 2.2 & 10.1 \\ D & 3.9 & 0.5 & - & -2.3 \\ E & 0.3 & 2.9 & -11.1 & -\end{array}$

Conformation a is in better agreement with NOE data than conformation b

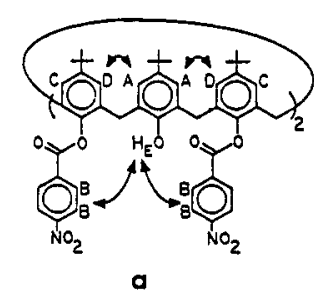

1,2,3-alternate

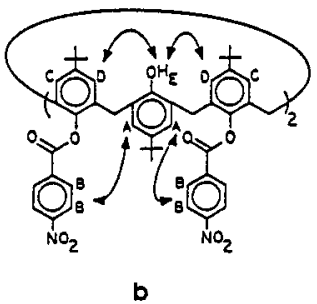

1,3,5-alternate

Oxyanions of Calix[6]arenes: Treatment of calix[6]arenes with incremental amounts of $\mathrm{n}$-butyllithium generates the mono-, di-, tri-, tetra-, penta-, and hexa-anions ${ }^{19}$ Temperature-dependent ${ }^{1} \mathrm{H}$ NMR studies of the hexa-anions from p-tert-butylcalix[6]arene and calix[6]arene show the latter to be somewhat more conformationally stable than the former. Treatment of calix[6]arenes with metal carbonates and acetates generates what are thought to be the tri-anions. The table below shows the coalescence temperatures and the free energies of activation for conformational inversion for $p$-tert-butylcalix[6]arene and calix[6]arene in the presence of various of these salts.

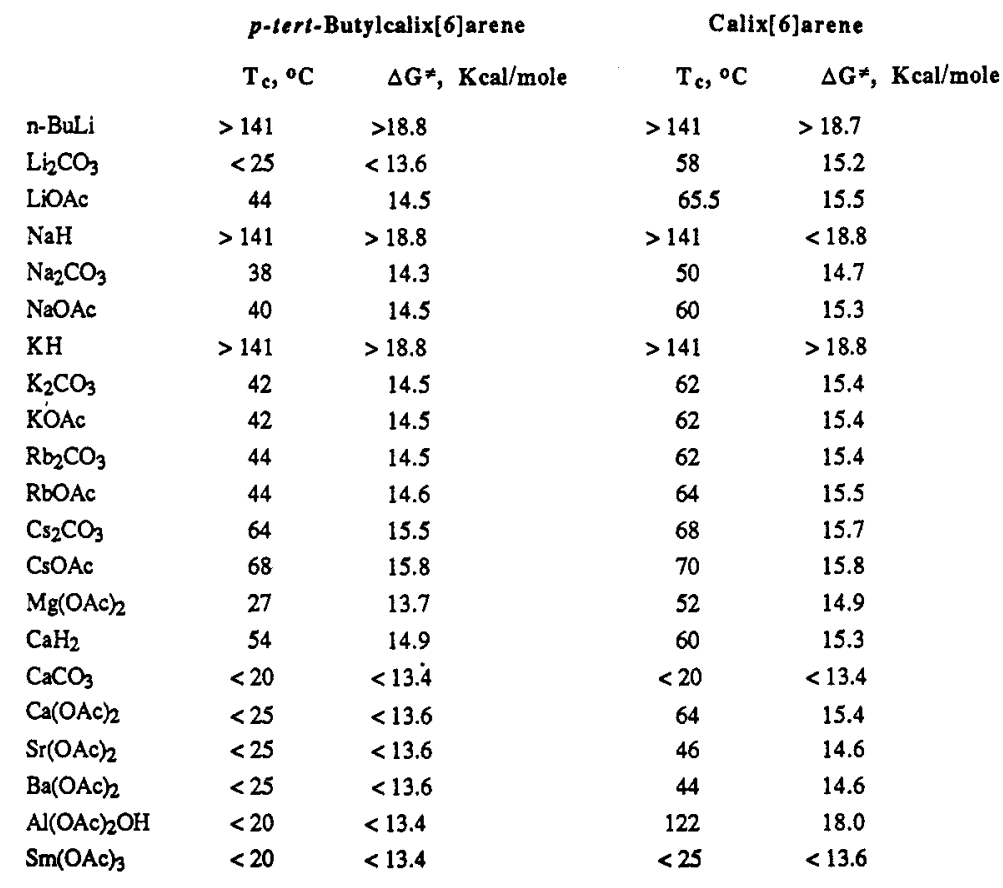

Acknowledgement We are indebted to the National Institutes of Health (Grant GM 23534), the National Science Foundation (Grant CHE 8611342) and the donors of The Petroleum Research Fund, administered by the American Chemical Society for their generous support of this work. 


\section{REFERENCES}

1 Thomas S. Kuhn, "The Structure of Scientific Revolutions", 2nd ed., The University of Chicago Press, Chicago, IL, 1962.

2 A. Baeyer, Ber., 1872, 5, 25, 280, 1094.

3 L. H. Baekeland, U. S. Patent 942,699 .

4 R. S. Buriks, A. R. Fauke, and J. H. Munch, U. S. Patents 4,032,514. 4,0998,717, and 4,259,464.

5 A. Zinke and E. Ziegler, Ber., 1941, B74, 1729.

6 J. B. Niederl and H. J. Vogel, J. Am. Chem. Soc., 1940, 62, 2512.

7 A. Zinke and E. Ziegler, Ber., 1944, 77, 264.

8 C. D. Gutsche and R. Muthukrishnan, J. Org. Chem., 1978, 43, 4905.

9 J. W. Cornforth, P. D'Arcy Hart, G. A. Nicholls, R. J. W. Rees, and J. A. Stock, Br. J. Pharmacol, 1955, $10,73$.

10 C. D. Gutsche, B. Dhawan, J. A. Levine, K. H. No, and L. J. Bauer, Tetrahedron, 1983, 39, 409.

11 H. Kämmerer, G. Happel, and F. Caesar, Makromol. Chem., 1972, 162, 179.

12 R. Muthukrishnan and C. D. Gutsche, J. Org. Chem., 1979, 44, 3962.

13 C. D. Gutsche, A. E. Gutsche, and A. I. Karaulov, J. Inclusion Phenom, 1985, 3, 447.

14 E. Paulus and V. Böhmer, Makromol. Chem., 1984, 185, 1921.

15 B. Dhawan, S.-I. Chen, and C. D. Gutsche, Makromol. Chem., 1987, 188, 921.

16 A. A. Moshfegh, E. Beladi, L. Radnia, A. S. Hosseini, S. Tofigh, and G. H. Hakimelahi, Helv. Chim. Acta, 1982, 65, 1264.

17 B. T. Hayes and R. F. Hunter, Chem. Ind., 1954, 193.

18 S. W. Bott, A. W. Coleman, and J. L. Atwood, J. Inclusion Phenom, 1987, 5, 747.

19 C. D. Gutsche, I. Alam, M. Iqbal, T. Mangiafico, K. C. Nam, J. Rogers, and K. A. See, J. Inclusion Phenom, 1989, 7, 61 . 